\title{
CYGNUS X-1: X-RAY EMISSION MECHANISM AND GEOMETRY
}

\author{
WEI CUI \\ MIT, Cambridge, MA 02139, USA \\ S. N. ZHANG \\ NASA/MSFC, Huntsville, AL 35812, USA \\ J. H. SWANK, X.-M. HUA AND K. EBISAWA \\ NASA/GSFC, Greenbelt, MD 20771, USA \\ AND \\ T. DOTANI \\ ISAS, Yoshinodai, Sagamihara, Kanagawa, 229, Japan
}

\section{Introduction}

On 1996 May 10, the All-Sky Monitor aboard RXTE revealed that Cyg X-1 started a transition from its hard state to soft state (Cui 1996). Throughout this interesting episode, snapshots were taken with more sensitive detectors on ASCA, RXTE, and CGRO to monitor the temporal and spectral variability of the source over a broad energy range.

The results (both spectral and temporal) from these observations seem to converge toward a self-consistent picture, which not only specifies the $\mathrm{X}$-ray emission geometry but also qualitatively describes how the geometry evolves during the transition.

\section{Results and Discussion}

The X-ray spectrum of Cyg X-1 extends beyond $100 \mathrm{keV}$ in both states. The spectral shape is rather complicated in details. At low energies, it is dominated by an ultra-soft component, which is thought to be the emission from an optically thick, geometrically thin disk. During the transition, the color temperature varies by a factor of $2-3$, and the luminosity (of this component) by a factor of 3 , indicating that the inner disk edge moves about 3 times closer to the black hole as the soft state is approached (Zhang et al. 
1997). At high energies the spectrum can be described by a Comptonized spectrum. It is steeper in the soft state, implying a smaller Comptonizing corona, perhaps due to more efficient cooling (Cui et al. 1997a). Such interpretation is strongly supported by the measured hard X-ray lags: much smaller in the soft state, as well as observed shape of power-density spectra (Cui et al. 1997a,c). The measured coherence function between various energy bands is nearly unity in both states (Vaughan \& Nowak 1997; Cui et al. 1997c), but is less during the transition (Cui et al. 1997c), implying that the corona indeed varies during such a period.

A Compton reflection "bump" is apparent on the spectrum, as well as the presence of an iron emission line. In the soft state, the reflecting medium is highly ionized and the solid angle subtended is smaller than in the hard state (Ebisawa et al. 1996; Cui et al. 1997b). This is consistent with the reflection occuring mostly at the inner disk edge that is closer to the black hole (thus hotter) in the soft state. The iron line centers at $\sim 6.4 \mathrm{keV}$ in the hard state, probably due to neutral irons (Ebisawa et al. 1996), while in the soft state it is mostly due to He-like irons (Cui et al. 1997b). This suggests that the line emission also originates in the innermost region of the disk. The broader line profile in the soft state supports this scenario.

Attempts have been made, for the first time, to simultaneously model the observed spectral and timing properties of Cyg X-1 (Hua et al. 1997). The model invokes an extended hot Comptonizing corona with a nonuniform density distribution. It reproduces the observed spectral shape well, and more importantly it is capable of explaining the frequency-dependence of measured hard lags, which has been a "dilema" for Compton models over the past decade (Miyamoto et al. 1988). It now becomes clear that such dependence is sensitive to the density distribution of the corona. Therefore, phase lag measurement may ultimately provide insights into the dynamics of hot corona. In the future, more physics need to be incorporated in the model to account for such features as the reflection bump and iron line.

\section{References}

Cui, W. 1996, IAUC. 6404

Cui, W., et al. 1997a, ApJ, 474, L57

Cui, W., Ebisawa, K., Dotani, T. \& Kubota, A. 1997b, ApJ, submitted

Cui, W., Zhang, S. N.,Focke, W., \& Swank, J. 1997c, ApJ, 484, 383

Ebisawa, K. et al. 1996, ApJ, 467, 419

Hua, X.-M., Kazanas, D., \& Cui, W. 1997, ApJ, submitted

Miyamoto, S., et al. 1988, Nature, 336, 450

Vaughan, B. A., \& Nowak, M. A. 1997, ApJ, 474, L43

Zhang, S. N., et al. 1997, ApJ, 477, L95 